\title{
Hemangioma del labio superior tratado con éxito con propranolol
}

\author{
C. Madrigal Díez ${ }^{\mathrm{a}}$, MR. Mazas Rabab ${ }^{\mathrm{b}}$, MI. Fernández Jiménez \\ EM. de Diego García ${ }^{c}$, R. Ballestero Diego ${ }^{d}$ \\ a Pediatra. CS Cazoña. Santander. España. \\ bediatra. CS Alto Asón. Cantabria. España. \\ ${ }^{C}$ Cirujano pediátrico. Hospital Universitario Marqués de Valdecilla. Santander. España. \\ ¿Urólogo. Hospital Universitario Marqués de Valdecilla. Santander. España.
}

\section{Resumen}

Los hemangiomas son los tumores benignos más frecuentes de la infancia, suelen localizarse en cabeza y tronco, y en la mayoría de los casos tienden a involucionar espontáneamente. Los hemangiomas labiales tienen mayor riesgo de producir deformidades, cicatrices residuales o ulceraciones. Una minoría de casos, bien por su tamaño, su ubicación o su evolución complicada, requieren tratamiento, empleándose tradicionalmente corticoides orales. En los últimos años han aparecido casos tratados con propranolol oral con excelentes resultados.

Presentamos un lactante varón de tres meses, sin antecedentes de interés, que presentaba un hemangioma en el labio superior que le dificultaba la succión y le provocaba una deformidad estética. Tras descartar patología asociada, a los cuatro meses comenzó tratamiento ambulatorio con propranolol oral en dosis inicial de 1,5 mg/ $\mathrm{kg} /$ día, con buenos resultados y sin complicaciones. La evolución fue satisfactoria, disminuyendo el tamaño y el color de la lesión.

Consideramos que el propranolol es una opción terapéutica eficaz y segura, y aunque son necesarios estudios clínicos amplios en los que el mecanismo de acción, la dosis y la duración del tratamiento queden aclarados, queremos destacar la figura del pediatra de Atención Primaria en la detección de posibles efectos adversos, en el seguimiento del paciente y en el apoyo a la familia.

Palabras clave: Labio. Hemangioma. Propranolol.

Hemangioma of the upper lip treated successfully with propranolol

Abstract

Hemangiomas are the most common benign tumours in childhood, usually located in the head and neck, and most of them suffer a spontaneous regression. Lip hemangiomas have a higher risk of causing deformities, residual scarring or ulceration. A minority of cases, either because of size, location or their complicated evolution, require therapy, traditionally using oral

Carmen Madrigal Díez, c.madrigaldiez@yahoo.es

Los autores declaran no presentar conflictos de intereses en relación con la preparación y publicación de este artículo. 
corticosteroids. In recent years, excellent results have been obtained in cases treated with oral propranolol.

We present the case of a 3-month-old male infant, with no history of interest, who had a hemangioma in his upper lip that made suction difficult and produced an aesthetic deformity. After ruling out associated pathology, at the age of 4 months our patient began outpatient treatment with oral propranolol with an initial dose of $1.5 \mathrm{mg} / \mathrm{kg} /$ day, with good results and without complications. The evolution was satisfactory, and the size and colour of the injury were reduced.

We believe that propranolol is an effective and safe therapeutic option, and although comprehensive clinical studies are needed in which the mechanism of action, dosage and duration of treatment are clarified, we would like to highlight the important role of the primary care pediatrician in the detection of possible adverse effects, in patient monitoring, and in family support.

Key words: Lip. Hemangioma. Propranolol.

\section{Introducción}

Los hemangiomas son los tumores vasculares más frecuentes de la infancia y suelen presentarse como lesiones solitarias en cabeza y cuello. Su evolución es difícil de predecir y no depende del tamaño ni de la localización de la lesión. La mayoría involucionan de manera espontánea en los primeros años de vida, de manera que la conducta a seguir es la observación y el control evolutivo. Sin embargo, en los casos de hemangiomas grandes, complicados o que producen afectación funcional, es necesario actuar, empleándose tradicionalmente los corticoides orales como primera opción terapéutica. En los últimos años han aparecido varias publicaciones de hemangiomas infantiles tratados con éxito con propranolol oral, con buena tolerancia clínica del paciente, y sin efectos colaterales, concluyendo que puede ser una alternativa eficaz y segura a los tratamientos utilizados hasta ahora.

Presentamos el caso de un lactante con un angioma en el labio superior que le dificultaba la succión y le producía deformidad estética, que presentó una buena y rápida respuesta al propranolol oral como tratamiento de primera elección.

\section{Caso clínico}

Varón fruto de un embarazo bien controlado y parto normal, con una exploración física al nacimiento normal salvo por un pequeño hemangioma cutáneo en el hipocondrio derecho. A los 45 días de vida presentó una mácula pálida en el labio superior que evolucionó a hemangioma. A los tres meses, la lesión dificultaba la succión y angustiaba a la familia por encontrarse en una localización muy visible (figura 1). De acuerdo con el Ser- 
vicio de Cirugía Pediátrica, y tras realizar un estudio ecográfico y un electrocardiograma, que fueron normales, se decidió iniciar tratamiento ambulatorio con propranolol a los cuatro meses de edad, debido al rápido crecimiento del hemangioma y sus posibles repercusiones estéticas y funcionales. La medicación se instauró en dosis de 1,5 mg/kg/día en una única toma. La respuesta al propranolol fue buena, estabilizándose el crecimiento de la lesión durante los primeros dos meses y disminuyendo progresivamente en los meses posteriores, por lo que se redujo la dosis a $1 \mathrm{mg} / \mathrm{kg} /$ día tras tres meses de tratamiento y se suspendió de- finitivamente a los 14 meses. La evolución del angioma del hipocondrio discurrió de forma paralela al del labio. Se realizaron controles periódicos de frecuencia cardiaca, tensión arterial y glucemia de forma ambulatoria, que se mantuvieron dentro de la normalidad. El paciente no sufrió efectos adversos graves, aunque los primeros meses mostró mayor inquietud y dificultad para conciliar el sueño, así como aplanamiento moderado de la curva ponderal, que se corrigió al disminuir la dosis de propranolol.

En la actualidad, con 17 meses, el niño se encuentra asintomático en un percen-

Figura 1. Hemangioma en el labio superior y hemangioma cutáneo en el hipocondrio derecho a los cuatro meses de edad.

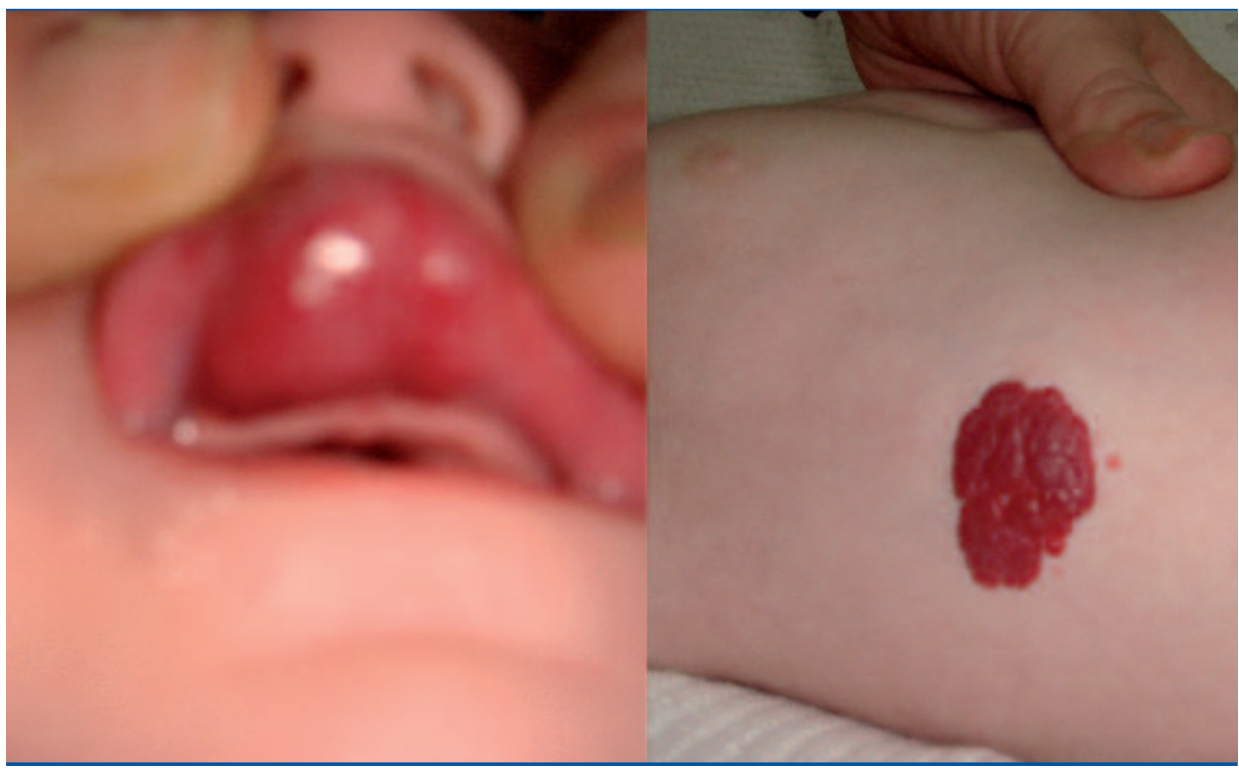


til de peso adecuado para su edad; el hemangioma no ha vuelto a aumentar de tamaño y no le produce ninguna repercusión funcional (figura 2).

\section{Discusión}

El manejo de los hemangiomas en la infancia continúa siendo una cuestión controvertida, porque no se dispone en la actualidad de una guía terapéutica realizada a partir de estudios rigurosos basados en la evidencia. Raramente es necesario tratar este tipo de lesiones por producir complicaciones serias o alteraciones estéticas o funcionales, y habitualmente suele ser suficiente con adoptar una actitud expectante mientras se observa su evolución natural.

Según las series publicadas, aproximadamente el 10-15\% de los hemangiomas requerirán un tratamiento activo, aunque solamente un $1 \%$ supone un verdadero peligro para la vida del paciente. Si bien en la actualidad existen diversos tratamientos, los corticoides sistémicos son aceptados generalmente como la primera opción terapéutica. Sin embargo, el uso de los corticoides en el tratamiento de los hemangiomas infantiles se basa en recomendaciones empíricas y su manejo varía entre médicos, existiendo pocos estudios sobre su efi-

Figura 2. Aspecto de las lesiones después del tratamiento con propanolol oral.

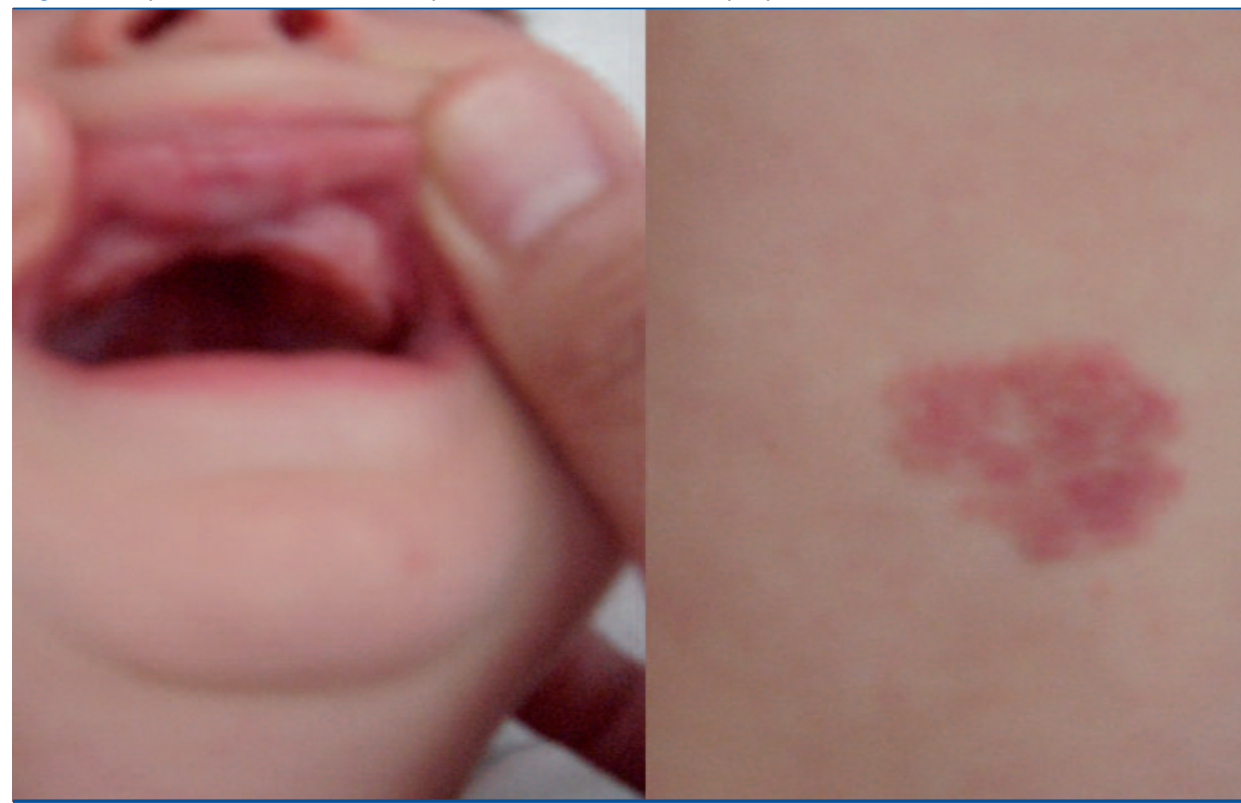


cacia real en el tratamiento de este tipo de lesiones, que además difieren en sus conclusiones. Por otra parte, existen hemangiomas agresivos que no responden a corticoides y pacientes a los que se les deben retirar por sus efectos secundarios. En estos casos, pueden emplearse medicaciones sistémicas alternativas como la vincristina o el interferón, pero tienen el inconveniente de ser costosas y producir efectos secundarios graves, sobre todo en lactantes.

En junio de 2008 se publicó el primer artículo que dio a conocer que el propranolol oral es capaz de inhibir el crecimiento de hemangiomas infantiles. Léaute-Labréze et al. ${ }^{1}$ observaron en dos pacientes, de manera accidental, una disminución evidente del tamaño y densidad de los hemangiomas cuando se les administraba propranolol por problemas cardiacos que habían surgido durante el tratamiento con dosis altas de corticoides sistémicos. Tras este hallazgo casual, administraron propranolol a nueve niños con hemangiomas capilares graves o desfigurantes, constatando una mejoría inmediata en el tamaño y el color de la lesiones sin presentar efectos adversos relevantes. En esta serie de pacientes, cuatro de ellos no habían respondido al tratamiento previo con prednisolona oral.
Desde que este artículo viera la luz, se han sucedido otros estudios observacionales que plantean que el propranolol puede ser una alternativa terapéutica eficaz y segura de los hemangiomas infantiles respecto a otros tratamientos habituales, pudiendo ser utilizado como primera línea de tratamiento. Sans et al. ${ }^{2}$, en su trabajo sobre el uso del propranolol en el tratamiento de hemangiomas infantiles graves, presentan una serie de 32 niños con una respuesta inmediata y eficaz a la administración oral de propranolol en dosis de $2-3 \mathrm{mg} / \mathrm{kg}$ diarios, que además mostraron una buena tolerancia clínica y escaso número de recidivas al suspender el fármaco. Sorprendentemente, el acortamiento de la fase de proliferación fue aún más notorio en los hemangiomas ulcerados, así como en aquellos que ocasionaban oclusión palpebral, compromiso hemodinámico o disnea en el paciente. Basel$\mathrm{ga}^{3}$ publica su experiencia en cinco pacientes con hemangiomas localizados en cabeza y cuello que fueron tratados con éxito con propranolol oral en dosis de $1,5 \mathrm{mg} / \mathrm{kg} / \mathrm{día}$, observando una mejoría inmediata del aspecto de las lesiones, que presentaron una disminución notable de su volumen y aclaramiento de su color en las 2-3 semanas siguientes. Ninguno de estos pacientes sufrió 
efectos secundarios graves. Excepto en un caso, los cuatro restantes habían sido tratados con corticoides sistémicos en dosis altas sin observarse respuesta.

Los efectos del propranolol en la regresión de los hemangiomas cutáneos se explica a través de varios mecanismos: en los hemangiomas produciría vasoconstricción (dando lugar a un rápido aclaramiento en el color y ablandamiento de la lesión al iniciar el tratamiento), menor expresión de los genes del factor de crecimiento del endotelio vascular (VEGF) y del factor de crecimiento fibroblástico básico (bFGF), mediante la regulación de la proteína quinasa activadora de mitogénesis (RAF), lo cual lleva a comprender la mejoría progresiva y sostenida del hemangioma y el inicio de la apoptosis de las células endoteliales de los capilares ${ }^{1}$.

Se ha descrito que los hemangiomas labiales evolucionan más lentamente, y se asocian a dificultades en la alimentación, salivación persistente y dificultad en la mordida. Además, tienen mayor riesgo de dejar deformidades o cicatrices residuales o ulceraciones ${ }^{4}$. En el caso que presentamos, la instauración temprana de un tratamiento que inhibiera la fase de proliferación del hemangioma del labio superior está justificada por las posibles secuelas estéticas, psicológicas y funcionales que podría presentar en el futuro nuestro pequeño paciente en el caso de una evolución desfavorable del mismo. Tras informar a los padres de la evolución espontánea impredecible de la lesión y las diferentes opciones terapéuticas disponibles, se decidió de forma consensuada comenzar con una dosis prudente de propranolol oral de $1,5 \mathrm{mg} / \mathrm{kg} /$ día, susceptible de ser aumentada dependiendo de la evolución de la lesión y de la aparición de efectos secundarios. No obstante, dada la buena respuesta clínica a las pocas semanas de iniciado el tratamiento, y teniendo en cuenta la ausencia de efectos secundarios graves, decidimos mantener la misma dosis durante tres meses para luego ir reduciendo paulatinamente la medicación.

La dosis máxima eficaz del propranolol está todavía por determinar, y el incremento de la dosis dependería de la gravedad de los síntomas y de la eficacia inicial del tratamiento ${ }^{5}$. Debido al número escaso de estudios observacionales publicados y a la ausencia de ensayos clínicos, aún no está bien establecido cómo y cuándo reducir la dosis. La duración del tratamiento depende de la edad del niño cuando se instaura el tratamiento y de la respuesta del paciente al mismo, aunque lo habitual es mantener la medicación por 
lo menos hasta que finaliza la fase de proliferación del hemangioma (hacia los 12 meses de edad), para evitar recidivas tempranas. La dosis debe reducirse de forma lenta para evitar la aparición de efectos adversos relacionados con una suspensión brusca del propranolol tras administraciones prolongadas, como taquicardia, nerviosismo y aumento de la tensión arterial.

El tratamiento con propranolol no está exento de riesgos, y el equipo médico que trata al niño debe conocerlos para intervenir de forma precoz en caso de que se produzcan. Los efectos adversos hemodinámicos más comunes son la bradicardia transitoria y la hipotensión, sin que se haya descrito ningún caso de muerte o enfermedad cardiovascular seria como consecuencia de una exposición directa a betabloqueantes tras más de 40 años de uso clínico en pacientes pediátricos. Otro efecto secundario importante que se debe tener en cuenta es la aparición de hipoglucemia, sobre todo en niños menores de tres meses y en las dos o tres horas siguientes a la toma del medicamento. El tratamiento con propranolol está contraindicado si existe enfermedad bronquial de base, ya que puede producir una reacción aguda del broncoespasmo en pacientes con historia previa de broncorreactividad, por lo que se debe investigar la existencia de antecedentes personales y familiares de atopia o crisis de broncoespasmo antes de iniciar el tratamiento ${ }^{6}$. En las series publicadas de niños con hemangiomas infantiles tratados con propranolol se han señalado otros efectos adversos leves y transitorios, como agitación e irritabilidad, alteración del sueño e insomnio, hiperhidrosis y diarrea.

En nuestro caso, los padres del niño refirieron un aumento de la inquietud motora y dificultad para conciliar el sueño nada más comenzar a tomar el propranolol, que se atenuaron al descender la dosis y desaparecieron en el momento en que se retiró la medicación. Por otro lado, aunque conservó el apetito, se produjo un enlentecimiento de la ganancia ponderal, que también recuperó en las semanas siguientes a la suspensión del fármaco.

Desde que se propuso por primera vez el propranolol como tratamiento de los hemangiomas infantiles, se han publicado varios casos en los que esta opción terapéutica se ha mostrado efectiva y segura. Aunque es necesario realizar en un futuro estudios clínicos controlados para evaluar su eficacia real y determinar la dosis óptima y la duración del tratamiento más oportuna, parece que el propranolol va a poder utilizarse de forma segura como primera 
línea terapéutica en los hemangiomas infantiles proliferativos, teniendo además la ventaja de ser un medicamento barato y de fácil acceso. Con la presentación de este caso pretendemos que el pediatra de Atención Primaria se familiarice con un tratamiento que, si bien ha de ser evaluado y controlado por un equipo multidisciplinario, debe acompañarse de unos controles periódicos frecuentes de vigilancia de aparición de posibles efectos adversos, que pueden perfectamente realizarse en el centro de salud tras llevar a cabo un estudio riguroso del paciente y descartar enfermedad bronquial o cardiaca.

\section{Bibliografía}

1. Léauté-Labréze $C$, Dumas de la Roque $E_{1}$ Hubiche T, Boralevi F, Thambo JB, Taïeb A. Propranolol for Severe Hemangiomas of Infancy. N Engl J Med. 2008;358:2649-51.

2. Sans V, Dumas de la Roque E, Berge J, Grenier N, Boralevi F, Mazereeuw-Hautier J et al. Propranolol for Severe Infantile Hemangiomas: Follow-Up Report. Pediatrics. 2009;124: e423-31.
3. Baselga E. Propranolol for the Treatment of Infantile Hemangiomas of Infancy. Pediatric Dermatology. 2008;25(6):674.

4. Zegpi MS, Sandoval M. Indicaciones quirúrgicas en hemangiomas de la infancia. A propósito de un caso. Rev Chilena Dermatol. 2008; 24(3):222-6.

5. López Gutiérrez JC. Propranolol y hemangiomas. An Pediatr (Barc). 2009;71:461.

6. Siegfried EC, Keenan WJ, Al-Juredini S. More on propranolol for Hemangiomas of Infancy. N Engl J Med. 2008;359:26. 